\title{
Esperienze operative presso un centro per le famiglie e dei nidi d'infanzia, attraverso l'approccio Touchpoints e il massaggio infantile
}

\author{
Elisabetta Ciracò*, Danila Grande** \\ *Psicologa, psicoterapeuta, insegnante AIMI, già professore a contratto, psicologia clinica e psicologia scolastica, \\ Università di Bologna, Dipartimento di Psicologia, sede di Cesena; \\ ** Educatrice, insegnante AIMI, consulente per I'allattamento, insegnante mini misp per il massaggio nelle scuole \\ dell'infanzia, educatrice presso i nidi del Comune di Roma
}

\begin{abstract}
Questo lavoro descrive l'esperienza operativa svolta con gruppi di genitori e bambini nei primi mille giorni di vita, che le autrici hanno realizzato negli anni nei servizi pubblici e nei centri per le famiglie, avendo come riferimento le indicazioni e i suggerimenti teorici di T.B. Brazelton e V. McClure. Entrambi presentano due approcci centrati sul sostegno e la promozione della relazione tra genitori, bambini e operatori. Tale lavoro evidenzia quanto i servizi territoriali, quali i centri per le famiglie e i nidi d'infanzia, mantengano da anni un ruolo essenziale e prioritario nel supporto alla genitorialità e favoriscano l'assestarsi dei delicati equilibri della rete familiare in un periodo di vita soggetto a forti vulnerabilità.
\end{abstract}

\section{This work describes the operational experience with groups of parents and children in the first 1000 days of life carried out over the years by the authors in public services and family centers based on the T.B Brazelton and V.McClure theoretical suggestions. Both present two approaches focused on supporting and promoting the relationship between parents, children and operators. The paper highlights how much local services such as Family Centers and Kindergartens have for years maintained an essential and priority role in parenting support and in stabilizing the delicate balance of the family network in a period of life subject to strong vulnerabilities.}

I started thinking that my work consisted in learning to appreciate parents. When I changed my attitude and started to trust them they began to trust more themselves.

Cominciai a pensare che il mio lavoro consisteva nell'imparare ad apprezzare i genitori. Quando cambiai il mio atteggiamento e iniziai a fidarmi di loro, loro cominciarono ad avere più fiducia in se stessi.

T. Berry Brazelton, "Learning to Listen. A Life Caring FOR Children", 2013

\section{Introduzione}

Le evidenze scientifiche concordano sull'importanza di investire sullo sviluppo precoce del bambino, per dare supporto nei primi mille giorni di vita e alle famiglie. Questo consente di promuovere il potenziale di salute e di sviluppo a breve e lungo termine dei bambini e ridurre e contrastare le disuguaglianze di salute.

L'idea che la storia biologica e comportamentale di un individuo derivi dal patrimonio genetico ereditato dai genitori appartiene ormai al passato. Lo insegna l'epigenetica, una maniera nuova di conside- rare il genoma, ovvero l'insieme di tutto il DNA e del patrimonio genetico di una specie. Essa studia il modo in cui l'ambiente interferisce sul genoma. Certamente lo sviluppo dell'uomo dipende dai geni, ma il loro funzionamento è fortemente influenzato dall'ambiente esterno. Quest'ultimo, infatti, condiziona i geni fin da prima del concepimento e continua a farlo durante la gravidanza e nei primi anni di vita del bimbo. Significa che gli stili di vita dei genitori, gli stimoli psicologici e le scelte intraprese prima e dopo la gestazione giocano un ruolo nello stato di salute del piccolo e futuro adulto [1].

È la cosiddetta "teoria dei mille giorni", nei quali si determina l'avvenire del bambino. In questa fase della vita, il cervello si sviluppa più che in qualsiasi altro momento e l'ambiente gioca un ruolo cruciale nel determinare la crescita e lo sviluppo futuro: le esperienze vissute nella prima infanzia, infatti, sono influenzate dal contesto in cui i bambini nascono e crescono e dalle figure adulte che per prime si prendono cura di loro. Esperienze positive precoci sono associate a migliori esiti scolastici, a un sano sviluppo sociale ed emotivo, a migliori risultati nel contesto lavorativo e, in generale, a un migliore stato di salute [2]. Come spiegano e confermano gli specialisti dell'Ospedale del Bambin Gesù di Roma (http://www.ospedalebambinogesu.it/ a-scuola-di-salute-i-primi-1000-giorni\#. XsVMCGgzaUm), nei primi mille giorni l'organismo è ancora in "via di costruzione", quindi è più plastico e malleabile.

Occorre sostenere e promuovere un "ambiente ottimale" (già dalla vita intrauterina) prendendosi cura della madre, del padre, della coppia genitoriale e del contesto in cui vive sia a livello micro (la famiglia, la comunità di appartenenza) che macro (la società, la cultura, le politiche, ecc). Lo sviluppo fisico, psichico e cognitivo del bambino può essere compromesso se un "ambiente ottimale" non viene tutelato e ci si trova in presenza di situazioni di "avversità" o di "carenza", come problemi di salute fisica o psichica dei caregiver, maltrattamento o violenza familiare, degrado ambientale e culturale, povertà economica e sociale. Investire nello sviluppo dei primi anni di vita (Early Child Development, ECD) favorisce lo sviluppo fisico e mentale del bambino con benefici che hanno un impatto durante tutta la vita.

Per questo motivo il tema deve essere prioritario nelle politiche e nei programmi, poiché è soprattutto in quegli anni che si gioca il benessere e la realizzazione delle generazioni future. Come riporta Marmot nella sua review del 2010 [3]: "Dare ad ogni bambino il miglior inizio di vita possibile è fondamentale per ridurre le disuguaglianze di salute nel corso della vita. Nella prima infanzia si creano le basi per ogni aspetto dello sviluppo umano, fisico, intellettuale ed emotivo. Ciò che accade in questi primi anni (a partire dalla vita intrauterina) ha effetti permanenti su molti aspetti della salute e del benessere, dall'obesità, le malattie cardiache e mentali ai 
risultati scolastici e allo status economico. Per avere un impatto sulle disuguaglianze di salute dobbiamo affrontare il gradiente sociale relativo all'accesso dei bambini a esperienze positive nei primi anni di vita. Gli interventi successivi, sebbene importanti, sono decisamente meno efficaci se mancano buone basi nella prima infanzia". Per questo, per esprimere il loro pieno potenziale, i bambini necessitano di cinque componenti della nurturing care:

1 buona salute;

2 nutrizione adeguata;

3 genitorialità responsiva;

4 opportunità di apprendimento precoce;

5 protezione/sicurezza.

Lo studio "First thousand days", è stato condotto dal Murdoch Childrens Research Institute di Sydney, che conduce e raccoglie ricerche sui fattori che influenzano lo sviluppo infantile, e mostra che le condizioni fisiche ed emotive in cui si viene al mondo influenzano le probabilità di esiti positivi o negativi durante la vita. La ricerca mostra che molti di questi cambiamenti avvengono prima ancora che il bambino sia concepito, e poi durante la gravidanza, arrivando alla conclusione che mentre i genitori svolgono un ruolo cruciale nello sviluppo di lungo termine del bambino, è necessario anche un ambiente positivo dentro l'intera comunità in cui la famiglia vive [5]. Ma questo sguardo attento al delicato periodo di vita del bambino e ai principi di cura e sostegno nella rete familiare e sociale, erano già presenti nei contributi dei due autori, T.B. Brazelton e V. McClure fin dagli anni Cinquanta.

\section{Obiettivi}

Vorremmo porre l'attenzione sui preziosi contributi che entrambi gli autori hanno fornito, Brazelton sul cambio di prospettiva sullo sviluppo del bambino e sulle dinamiche relazionali genitori-bambini-operatore e McClure, sulla pratica e l'efficacia dell'operatività del massaggio infantile. Nello specifico:

- rilevare gli apporti nella pratica: la funzione dell'operatore e i principi;

- riportare la narrazione delle esperienze: laboratori di massaggio infantile e nel nido.

\section{Apporti nella pratica: Ia funzione dell'operatore e i principi secondo Brazelton e McClure}

L'operatore formato nell'approccio Touchpoints di T.B. Brazelton può esser considerato come colui che porta "buoni semi" di conoscenza al genitore, impegnato nella costruzione della relazione con il figlio. In seguito alla formazione egli acquisisce modo di essere e di agire e questa "impalcatura formativa" è il fondamento di un incontro nuovo e della possibilità di una partnership genitore-operatore. Le famiglie sono il primo ambiente relazionale di un piccolo essere umano e hanno un ruolo fondante nella costruzione del sistema cerebrale.

L'operatore formato nell'approccio Touchpoints, sia in ambito sanitario, che psicologico e educativo può, come ci ricorda Bronfenbrenner [6] nel suo approccio ecologico al sistema relazionale, essere colui che manifesta ammirazione e affetto per le figure genitoriali che per prime si prendono cura del bambino. Un'alleanza fra operatori e genitori può tradursi in interazioni con il bambino più stimolanti. L'approccio Touchpoints rivaluta e riformula le tradizionali impostazioni sia in ambito medico sia in ambito educativo, modificandone l'agire. I cambi di prospettiva riguardano lo spostamento del focus dal possibile deficit alle caratteristiche positive; concentrarsi sulle competenze restituisce al genitore fiducia rispetto alle sfide della crescita. A1 genitore viene restituito un ruolo primario di valutazione rispetto alla crescita e l'operatore perde il ruolo prescrittivo che aveva. Il coinvolgimento empatico tra l'operatore e le famiglie favorisce l'ascolto attivo e un rapporto di vera collaborazione per il bene comune. Secondo l'approccio Touchpoints l'operatore viene aiutato nel formare l'alleanza con il genitore utilizzando alcuni princìpi di base, tra cui i fondamentali sono quelli di: usare il comportamento del bambino come proprio linguaggio; cercare occasioni per sostenere le competenze dei genitori; valorizzare la passione e le emozioni ogni qual volta si incontrano; valorizzare la disorganizzazione.

L'altro strumento che l'approccio Touchpoints propone per avvicinarsi alla prospettiva del genitore e del bambino, e quindi a sostenerne la relazione, è quello di utilizzare dei presupposti, cioè degli assunti che aiutano il modo di porsi dell'operatore verso i genitori, tra cui ritenere che i genitori sono gli esperti del loro bambino (gli operatori lo sono dei bambini in generale), che hanno sempre dei punti di forza, che vogliono fare il meglio per il loro bambino, che possono avere dei sentimenti ambivalenti verso di lui e che vivono momenti critici e sentono il bisogno di condividerli [7].

\section{Princìpi guida secondo McClure}

La relazione madre-bambino è un sistema di regolazione reciproca. La qualità dello scambio affettivo gioca un ruolo fondante nello sviluppo sociale ed emozionale del bambino. Madre e figlio sco- prono i modi dello stare assieme, cioè partecipano alla creazione dei significati diadici. Il massaggio infantile rappresenta uno strumento prezioso per affinare questo dialogo. L'operatore promuove la buona interazione genitore-figlio proponendosi come facilitatore della relazione, accompagnandolo attraverso l'ascolto attivo nello sfidante terreno dell'incontro col figlio. Attraverso la riformulazione, le domande aperte, le esperienze e i sentimenti dei genitori che vengono accolti e non negati; essi vengono sostenuti a dar voce al proprio intuito e a proporre suggerimenti che siano in linea col loro pensiero. Incoraggiarli a cercare il contatto col figlio e a rispondere ai segnali comunicativi, partendo dal riconoscimento degli stati comportamentali, facendo del comportamento del bambino il proprio linguaggio. Insegnare il rispetto, con la richiesta del permesso, giacché il massaggio è un dialogo che si sviluppa con il bambino. Lo strumento realizzato si è sviluppato nell'esperienza pratica delle autrici in ambiti operativi territoriali differenti (Emilia-Romagna e Lazio) quali il centro per le famiglie del territorio romagnolo e il servizio infanzia del Comune di Roma.

\section{Metodo e strumenti presso il centro per} le famiglie del territorio romagnolo

L'esperienza presso il centro per le famiglie della Romagna forlivese sviluppata in diciotto anni di attività (dal $2000 \mathrm{al}$ 2018) con conduzione di gruppi di massaggio, è stata descritta in un articolo [8] e in due lavori presentati come "Poster" nel 2019 all'assemblea nazionale dei trent'anni dell'AIMI a Bologna e all'incontro della rete nazionale Touchpoints a Roma. La ricerca è stata condotta a partire dall'individuazione dei corsi di massaggio infantile organizzati nelle strutture del territorio romagnolo presso il centro per le famiglie della Romagna forlivese (coordinato dallo psicologo Fabio Canini). Tali corsi, strutturati secondo le indicazioni della formazione AIMI, si sono articolano in 4/5 incontri della durata di un'ora e mezza, rivolti ai neogenitori e ai loro bambini entro il primo anno di vita. Tutti gli incontri ruotano intorno a contenuti teorici relativi allo sviluppo del bambino (benessere fisico e psicologico, ruolo genitoriale, potenzialità del massaggio infantile) e contemplano momenti interattivi e pratici, nei quali ciascuno può sperimentare concretamente la pratica del massaggio e la riflessione condivisa in gruppo di genitori.

L'approccio nel massaggio AIMI sostiene la relazione attraverso l'accoglienza, l'accettazione, il rispetto (es. richiesta ini- 
ziale del permesso) e la discontinuità dello sviluppo e il massaggio lavora principalmente intorno a quattro grandi aree: stimolazione, sollievo, rilassamento e interazione [9]. Utilizzando i segnali comportamentali del bambino ne decodifica e legge gli stadi, come anticipazione di successivi passaggi di maturazione e di crescita. Nella cornice delle modalità di cura positive e determinanti per la "costruzione" delle basi neurobiologiche del bambino, si inserisce la pratica del massaggio infantile neonatale, considerato uno degli strumenti a disposizione dei genitori per facilitare il rapporto e la comunicazione con il proprio figlio [10-11]. Di seguito vengono definiti i benefici del massaggio infantile, nell'esperienza di madre e di padre (Tabella 1) [12-13], nella relazione famigliare (Tabella 2) e quali le ricadute nella comunità sociale (Tabella 3) e gli effetti benefici per il bambino [14].

I servizi sanitari territoriali, i consultori pubblici della neuropsichiatria infantile e i centri per le famiglie si sono attivati, nel tentativo di allinearsi alle "Buone pratiche costruttive dello sviluppo nei primi 1000 giorni di vita” (Regione Emilia-Romagna, Bologna, 10 maggio 2018, Giorgio Tamburlini) e di trasformarsi in veri e propri luoghi di accoglienza e supporto, nei quali accompagnare gli adulti nel costruire un legame sensibile e adeguato con i loro bambini.

La ricerca è stata condotta a partire dall'individuazione dei corsi di massaggio infantile organizzati nelle strutture del territorio romagnolo presso il centro per le famiglie della Romagna forlivese. I laboratori si sono svolti in $4 / 5$ incontri e sono stati rivolti ai neogenitori e ai loro bambini entro il primo anno di vita. I contenuti teorici sono stati rivolti allo sviluppo del bambino, al suo benessere fisico e psicologico, alla centralità del ruolo genitoriale, alle potenzialità del massaggio infantile. Favoriti, inoltre, momenti interattivi, volti alla raccolta di bisogni specifici, esigenze, domande, e momenti pratici, nei quali ciascuno ha potuto sperimentare concretamente la pratica del massaggio. In occasione di tali corsi è stato distribuito a tutti i partecipanti un questionario costruito ad hoc, volto ad esplorare le percezioni e il grado di soddisfazione relativo all'andamento dei corsi.

Dei 752 iscritti che hanno partecipato ai corsi, hanno consegnato il questionario 503 neogenitori, di cui 499 madri e 4 padri (madri $=99,2 \%$; padri $=0,8 \%)$. È stata realizzata un'analisi descrittiva sul contenuto delle risposte, mettendo in evidenza la frequenza e la tipologia delle tematiche riportate a partire da quanto emerso

TABELLA 1. Benefici del massaggio per i genitori

\begin{tabular}{|l|l|}
\hline MADRE & PADRE \\
\hline Riduce il rischio di maternity blues & Diminuisce il timore di esclusione \\
\hline Favorisce benessere e rilassamento & Favorisce la competenza genitoriale \\
\hline Incrementa la cura & Incrementa la vicinanza con madre e bambino \\
\hline
\end{tabular}

TABELLA 2. Benefici del massaggio per la famiglia

II linguaggio della pelle promuove la relazione fra fratelli

Contiene il senso di gelosia

Accoglie le specificità

TABELLA 3. Benefici del massaggio per la società

Crescita di una cultura dell'infanzia guidata da rispetto ed empatia

Riduzione dell'incidenza di maternity blues

Protezione dal rischio di abuso infantile

Prevenzione e promozione della salute basato sulle life skills (o competenze per la vita), secondo indicazione OMS, necessari per affrontare le sfide della vita

nei questionari. Obiettivi dell'analisi sono stati quelli di: indagare il grado di soddisfazione e le aspettative relative alla partecipazione al corso di massaggio dei neogenitori; indagare la correlazione tra caratteristiche specifiche del corso (es. numero di incontri, contenuti affrontati, ecc.) e utilità percepita dai partecipanti; esplorare i bisogni e le motivazioni dei genitori; raccogliere suggerimenti relativi allo svolgimento del corso e/o a proposte ulteriori destinate ai neogenitori e ai loro figli.

I risultati hanno evidenziano un'elevata soddisfazione (98\%) dei neogenitori, in quanto i corsi favoriscono uno spazio di relazione e di tempo privilegiato con il proprio figlio, oltreché l'acquisizione di una tecnica utile al benessere di entrambi. Emerge inoltre il bisogno di creazione di una rete sociale attiva tra genitori nella stessa fase di vita e di confronto con professionisti, cui affidare dubbi e fragilità. Tali elementi sono rimasti invariati nel corso di 18 anni di esperienza, a dimostrazione di quanto i servizi territoriali siano interlocutori indispensabili nella fase delicata di transizione alla genitorialità. Tale lavoro evidenzia quanto i servizi territoriali, quali i centri per le famiglie, mantengano da anni un ruolo essenziale e prioritario nel supporto alla genitorialità (secondo le linee ministeriali sui "Primi 1000 giorni di vita”), e in particolare nella prevenzione primaria.

\section{Metodo e strumenti presso un servizio} infanzia del Comune di Roma

Lo strumento realizzato si è sviluppato nell'esperienza pratica in un nido del Comune di Roma. Brazelton diceva che quando rafforziamo le famiglie, in sinte- si, rafforziamo la comunità. L'esigenza di essere supportati da operatori consapevoli e competenti, che sappiano sviluppare fiducia nelle capacità genitoriali, risponde ai bisogni irrinunciabili dei bambini; inoltre aiuta nello sviluppare relazioni e nel dare protezione, facendo vivere esperienze a misura delle specificità, con i giusti limiti, e proteggendo il futuro.

McClure ci ricorda come il toccare, il sentire e il memorizzare con la pelle ci mettono alla ricerca di legami e di sintonie col mondo e sono in grado di sostenere lo sviluppo del sé. È evidente come i due studiosi abbiano in comune uno sguardo sulla relazione genitore-bambino che immagina lo sviluppo come un processo evolutivo discontinuo e in sincronia sul modello relazionale, con il focus sull'alleanza educativa fra genitore e operatore, elemento sostenitore del processo di crescita.

Stare al nido è un progetto fatto di alleanza, che vede genitori e educatori, coprotagonisti di un percorso che ha come obiettivo il benessere delle famiglie. Che cosa è allora l'alleanza? È un patto la cui essenza consiste nel far nostro come educatori, il trasporto che i genitori provano per il proprio figlio e tutti quei sentimenti a esso correlati, inclusa l'ambivalenza di emozioni per questa responsabilità e i timori legati alla crescita. Noi accogliamo tutto questo e ce ne prendiamo cura.

L'alleanza educativa ha bisogno di ascolto empatico e di sospensione del giudizio (vera e propria "palude" nell'incontro col genitore). Ciò che deve sostenerci è la competenza sullo sviluppo del bambino. Da questa deriva il modo in cui entriamo in rapporto coi genitori e la possibilità di usare il comportamento del bambino co- 
me nostro linguaggio. La genitorialità è un processo di pancia, di testa, di cuore, costruito su prove ed errori.

Nell'ottica Touchpoints valorizzare e comprendere la relazione fra noi e il genitore ha come assunto di base la convinzione che ogni genitore possieda punti di forza e sia il migliore esperto del proprio figlio, che desideri il meglio per lui pur provando anche sentimenti di ambivalenza che deve poter condividere. Stare al nido significa in questa dimensione di pensiero, costruire assieme un sistema di caregiving condiviso, consapevoli della responsabilità verso le generazioni future; creare una comunità competente e solidale che sarà in grado di sostenere in prospettiva la capacità dei bambini di provvedere alle loro stesse future famiglie e in una prospettiva più ampia, alla stabilità economica, politica e sociale di un Paese.

Il nostro nido è pensato non solo come spazio di ricerca e di sperimentazione ma anche come luogo dove i genitori possono restare ogni volta che vogliono per vivere assieme esperienze e momenti affettivamente nutrienti in un tempo lento e a misura di tutti. Gli educatori possono confrontarsi coi genitori e insieme, da meri fornitori d'informazioni, possono trasformarsi in moltiplicatori delle capacità che il bambino già possiede nei suoi codici.

La sperimentazione di questa impostazione metodologica è durata due anni in un primo nido ed è proseguita nella nuova piccola sede che accoglie solo bambini due-tre anni. Con il gruppo di educatrici precedente abbiamo programmato un sistema di accoglienza e ambientamento, organizzato in rete, che si attiva dal periodo estivo (dove siamo a casa ma "a disposizione” dal punto di vista lavorativo) coinvolgendo i nuovi iscritti in una sorta di home visiting virtuale. Abbiamo chiesto alle famiglie di prendere contatto fra di loro e inviato un'email di presentazione che spiega la nostra idea di nido; ai genitori abbiamo chiesto, in sostituzione delle vecchie schede informative, di raccontarci la loro famiglia. Abbiamo curato poi la preparazione degli spazi d'identità nella sezione (foto, nomi), consapevoli del valore dell'essere "ri-conosciuti" e dell'accogliere l'identità del gruppo.

Lo scambio di esperienze tra famiglie, che sono state accolte tutte insieme durante l'ambientamento iniziale ha fornito rassicurazione, senso di appartenenza e ci ha permesso di osservare da subito gli stili comunicativi e relazionali genitore-figlio ponendo le basi per il fare futuro. Questo stile empatico si è rivelato fortemente efficace con i genitori e anche per questo, quando sono stati invitati a giocare con gli stessi materiali usati dai bimbi cercando di contattare le loro emozioni, lo hanno fatto con gioia e trasporto. Sempre ai genitori è stato chiesto (invece che per decisione dell'educatore come avviene di solito), di capire quando era il momento per allontanarsi durante l'ambientamento e lasciare i bambini alle educatrici. La decisione è stata presa osservando i comportamenti dei bambini e trasformandoli in veri e propri linguaggi.

I genitori, fuori dalla porta, erano pieni di sensazioni, pensieri che andavano accolti e lo abbiamo fatto raccogliendoli nella "scatola dei pensieri". Le emozioni della coppia genitoriale sono parte integrante del percorso al nido, perché qui entra una famiglia e non un singolo bambino; per questo è stato loro offerto uno spazio dedicato ad ascoltarli. L'idea è di lasciare spazio ai loro vissuti sia come singoli che come coppie offrendo laboratori sull'ascolto attivo, sulla difficoltà nella coppia e utilizzando canali giocosi come l'arte-terapia.

Sempre in quello spazio hanno potuto trascorrere del tempo con il figlio attraverso il massaggio o cantando con lui e per lui. In questo modo la memoria tattile di adulti e bambini resta attiva. Le carezze che partono dal cervello arrivano all'anima e i figli potranno diventare futuri adulti che sapranno prendersi cura dell'altro. Sapranno restituire le carezze che hanno imparato attraverso i sensi. Il genitore potrà "decentrarsi”, pur mantenendo il sostegno, dall'accompagnare e lasciare andare.

Mettere in luce la sostanza della relazione appassionata che unisce noi e le famiglie è stato ed è un dono prezioso; insieme abbiamo costruito "impalcature" affettive che i responsabili dei servizi hanno il dovere di promuovere e legittimare. Brazelton ci ha parlato dei bisogni dei bambini nello stesso modo in cui ha sottolineato quelli degli adulti assieme alla necessità di essere entrambi accolti in una comunità che sappia "fare da guardia". Se infatti la "diade" genitoriale non viene aiutata a riflettere sui suoi bisogni e non ottiene sostegno in questo senso, vive male il proprio ruolo.

La situazione relazionale fra famiglie e educatori nel tempo rischia di rimanere imbrigliata in situazioni di competizione e rivalità. Anche gli educatori o insegnanti, se hanno bisogni non ascoltati, tendono a generare situazioni di antagonismo giudicante. Il lavoro educativo ha veramente senso se si costruisce come un cerchio: si parte dal lavoro su se stessi (l'educatore) per poi prendersi cura dell'altro e del modo in cui stiamo con lui, affinando lo sguar- do, in una costruzione resiliente di saperi condivisi.

Interventi a supporto della genitorialità I laboratori a supporto della genitorialità sono stati organizzati nella sezione dei piccolissimi, dei medi e dei grandi (questi ultimi nel micronido dove attualmente presto servizio). Il massaggio nella sezione dei piccolissimi è stato introdotto per consolidare la relazione all'interno del nido come risposta al bisogno irrinunciabile di bambini e famiglie di sviluppare costanti relazioni di accudimento.

Abbiamo organizzato sessioni di massaggio settimanali per cinque settimane, con gruppi di tre mamme con i propri figli all'interno della sezione subito dopo il sonno pomeridiano. Su un gruppo totale di quindici bambini iscritti alla sezione, hanno partecipato dodici mamme con i piccoli.

Per quanto riguarda il sostegno alla coppia genitoriale abbiamo organizzato due laboratori: il primo - che guarda alla "relazione come rel-azione", ovvero come fonte di consapevolezza e responsabilità dei propri sentimenti, come genitore, $\mathrm{CO}^{-}$ me coppia e come persona - è stato condotto da una psicoterapeuta (Daniela Di Renzo) e hanno partecipato la totalità degli iscritti alla sezione. Il secondo laboratorio è stato inserito nel percorso di alfabetizzazione musicale secondo la "music learning theory" di Edwin Gordon, ed è stato seguito da tutti i bambini insieme ai genitori in tutti e tre gli anni. Inoltre, abbiamo organizzato l'incontro "Vuoi cantare con me? Avventure musicali alla scoperta del proprio mondo interiore e di quello dei propri figli" condotto dalla counselour Francesca Scaglione Art, pianista e cantante, a cui hanno partecipato tutti i genitori.

Nella sezione dei medi, sono stati organizzati laboratori esperienziali di gioco genitori-figli con materiali naturali e di riciclo. Hanno partecipato, divisi in gruppi, tutti i genitori.

Nel nuovo micronido tutti i genitori sono stati accolti attraverso piccoli laboratori di costruzione di oggetti transizionali per i propri figli durante il periodo dell'ambientamento. Nel periodo natalizio tutti i genitori hanno partecipato a un incontro dove hanno creato con materiali di riciclo e foto dal forte significato affettivo per il figlio, un piccolo "album degli affetti".

La partecipazione delle famiglie agli incontri proposti durante la frequenza al nido ha rafforzato nel gruppo educativo la valenza di questa impostazione metodologica. Il "fare educativo" realizzato nell'ambiente nido ha contribuito a dare una dimensione 
comunitaria, in cui gli operatori hanno stabilito patti di alleanza fatti di buone pratiche con i genitori. In questa prospettiva le famiglie sono diventate luoghi di rielaborazione creativa degli stimoli proposti dalle educatrici in un arricchimento reciproco.

\section{Conclusioni}

Mai come in questo periodo storico, il ventunesimo secolo travolti dalla pandemia, il contatto, le relazioni e il prendersi cura sono diventati i temi emergenti e fondamentali per la salvaguardia della crescita e del benessere dei piccoli, dei genitori e della comunità. Le esperienze descritte rappresentano percorsi concreti e fattibili per offrire supporto alle famiglie e ai bambini nei primi mille giorni di vita promuovendo il potenziale di salute e sviluppo e per ridurre e contrastare le disuguaglianze di salute. In entrambi le sedi operative, seppur diversi per contesto e luogo geografico, emerge con forza il bisogno di una rete sociale attiva tra genitori nella stessa fase di vita e di confronto con professionisti, cui affidare dubbi e fragilità. L'obiettivo è costruire assieme un sistema di caregiving condiviso, consapevole della responsabilità verso le generazioni future per creare una comunità competente e solidale in grado di sostenere in prospettiva la capacità dei bambini di provvedere alle loro future famiglie e, in una prospettiva più ampia, alla stabilità economica, politica e sociale di un Paese.

\section{\luisaciraco@gmail.com; danila.grande@libero.it}

1. Burgio E. Dalla genetica all'Epigenetica. Soma Istituto Milano, 2019.

2. Prevenire è prendersi cura: un focus sugli interventi di Early Child Development. Euro Health Net Magazine. https://eurohealthnet-magazine.eu/prevenire-e-prendersi-cura-un-focus-sugli-interventi-di-early-child-development/.

3. UCL Institute of Health Equity. Fair Society, Healthy Lives: Strategic Review of Health Inequalities in England post-2010. The Marmot Review. February 2010. http://www.parliament.uk/documents/fair-society-healthylives-full-report.pdf

4. Brazelton TB. Learning to Listen. A Life Caring for Children. 2013.

5. Primi 1000 giorni di vita i più importanti per lo sviluppo. Sin dal concepimento si preparano basi salute fisica e mentale. Ansa-Sydney 26 settembre 2017.
6. Bronfenbrenner U. Making Human Beings Human. Bioecological Perspectives on Human Development. Thousand Oaks, 2005.

7. Brazelton TB, Greenspaan SI. I bisogni irrinunciabili dei bambini. Raffaello Cortina Editore, 2001.

8. Addarii F, Ciracò E, Polo K, et al. Uno studio descrittivo su 18 anni di attività presso centri per le famiglie del territorio romagnolo. Quaderni acp 2020;2.

9. McClure V. Massaggio al bambino, messaggio d'amore: Manuale pratico di massaggio infantile per genitori. Bonomi editore, 2014.

10. Guzzetta A, Baldini S, Bancale A, et al. Massage accelerates brain development and the maturation of visual function. J Neurosci. 2009 May 6;29(18):6042-51.

11. Guzzetta A, D’Acunto MG, Carotenuto $\mathrm{M}$, et al. The effects of preterm infant massage on brain electrical activity. Dev Med Child Neurol. 2011 Sep;53 Suppl 4:46-51.

12. Cullen C, Field T, Escalona A, et al. Father-infants interactions are enhanced by massage therapy. Early Child Development and Care. 2000;164(1): 41-7.

13. Gürol A, Polat S. The Effects of Baby Massage on Attachment between Mother and their Infants. Asian Nurs Res (Korean Soc Nurs Sci). 2012 Mar;6(1):35-41.

14. Lucangeli D. V Convegno: le mani che nutrono. L'educazione al Contatto per una società empatica, Padova. 2019.

\section{La chirurgia nei bambini neurologici è a più alto rischio di polmonite post intervento}

Anche i bambini affetti da patologie neurologiche possono avere bisogno - come tutti i bambini e forse più degli altri - di un intervento chirurgico. Qualsiasi procedura di questo tipo ci appare ragionevolmente a più alto rischio durante, ma anche successivamente all'atto chirurgico in sé. La possibilità di sviluppare un'infezione respiratoria postoperatoria è concreta ed è favorita da scarsa coordinazione neuromuscolare, tosse inefficace, incoordinazione della deglutizione e insufficiente igiene orale. Una ricerca retrospettiva USA, condotta su scala nazionale, ha cercato di quantificare il rischio di polmonite postoperatoria in bambini con comorbilità neurologica, prendendo in esame una casistica di 349.163 bambini dei quali $2.191(0,6 \%)$ avevano sviluppato un'infezione respiratoria entro 30 giorni dall'intervento. La presenza di una patologia neurologica conferiva un rischio circa due volte maggiore di polmonite postoperatoria (hazard ratio [HR]: 1,91). All'interno di questa popolazione, i bambini con paralisi cerebrale avevano il rischio più elevato (HR: 3,92), seguiti da quelli con epilessia (HR: 2,93) e con patologia neuromuscolare (HR: 2,63). La presenza di una comorbilità neurologica determinava anche una maggiore durata della degenza in ospedale.

I timori di chiunque di noi accompagni uno di questi bambini all'intervento chirurgico sembrano, quindi, fondati. I dati, provenienti da diverse istituzioni statunitensi non consentono ulteriori approfondimenti, ma la raccomandazione che emerge è di elaborare strategie preventive delle polmoniti postoperatorie in tutti questi pazienti. Gli autori citano l'efficacia della pulizia dentale con spazzolatura e clorexidina per ridurre la contaminazione del cavo orale, ma forse dovremmo puntare anche su un'adeguata fisioterapia respiratoria e mobilizzazione che realizzi un'efficace drenaggio delle secrezioni bronchiali. Nulla di tutto ciò è provato al di là di ogni ragionevole dubbio, ma ogni ulteriore attenzione può rivelarsi utile per ridurre il rischio aggiuntivo di ulteriori complicanze.

1. Mpody C, Hayes S, Rusin N, et al. Risk Assessment for Postoperative Pneumonia in Children Living With Neurologic Impairments. Pediatrics. 2021 Aug 4;e2021050130. 